\title{
Successful treatment of rectal cancer with perineal invasion: Three case reports
}

\author{
TOMOHIRO KITAHARA ${ }^{1}$, MAMORU UEMURA ${ }^{1}$, NAOTSUGU HARAGUCHI ${ }^{2}$, JUNICHI NISHIMURA ${ }^{1}$, \\ TATSUSHI SHINGAI ${ }^{3}$, TAISHI HATA ${ }^{1}$, ICHIRO TAKEMASA ${ }^{1}$, TSUNEKAZU MIZUSHIMA ${ }^{1}$, \\ YUICHIRO DOKI $^{1}$, MASAKI MORI $^{1}$ and HIROFUMI YAMAMOTO ${ }^{1}$ \\ ${ }^{1}$ Department of Gastroenterological Surgery, Graduate School of Medicine, Osaka University, Suita, Osaka 565-0871; \\ ${ }^{2}$ Department of Gastroenterological Surgery, National Hospital Organization, Osaka National Hospital, Osaka, \\ Osaka 540-0006; ${ }^{3}$ Department of Surgery, Saiseikai Senri Hospital, Suita, Osaka 565-0862, Japan
}

Received December 1, 2013; Accepted February 24, 2014

DOI: $10.3892 / \mathrm{mco} .2014 .282$

\begin{abstract}
Rectal cancer occasionally invades adjacent organs. However, rectal cancer with perineal invasion is uncommon and difficult to treat. Locally advanced colorectal cancer may be clinically treated with neoadjuvant therapy, followed by en bloc resection. Skin invasion may lead to tumor dissemination via cutaneous blood flow and lymphatic routes. There is currently no firm evidence regarding the treatment of these significantly advanced rectal cancers. In this study, we report 3 cases of rectal cancer with perineal invasion, successfully managed by multimodality treatment. Case 1 is a 52 -year-old man with rectal cancer that had invaded the perineum; case 2 is a 38-year-old man with rectal cancer infiltrating the perineal skin and liver metastasis; and case 3 is a 50 -year-old woman with rectal cancer and perineal invasion. All the cases were treated with radical excision. No severe complications were observed in the perioperative period. Case 2, in particular, was confirmed to remain alive 5 years after the surgery. Our experience suggests that multimodality treatment, including extended radical surgery, may be a feasible approach to the treatment of rectal cancer with perineal skin invasion.
\end{abstract}

\section{Introduction}

Colorectal cancer most often disseminates to the liver and lung and rarely to the skin $(1,2)$. The incidence of skin involvement for colorectal cancer is reported as low as $2.3 \%$ (3). Cutaneous metastases from colorectal cancer are generally caused by direct tumor extension, dissemination, invasion of the embryonic ligaments, or implantation during surgical resection (4).

Correspondence to: Dr Hirofumi Yamamoto, Department of Gastroenterological Surgery, Graduate School of Medicine, Osaka University, 2-2 Yamada-oka, Suita, Osaka 565-0871, Japan

E-mail: hyamamoto@gesurg.med.osaka-u.ac.jp

Key words: rectal cancer, perineal invasion, multimodality treatment, extended radical surgery
Massive invasion of rectal cancer to the perineal skin is considered extremely rare, since the sensation of a perianal lump, altered bowel habits, bleeding or soiling, usually leads to diagnosis prior to the occurrence of massive invasion to the perineal area (5). Patients with perineal skin invasion by rectal cancer present with symptoms of severe pain, continuous bleeding and distressing discharge, which significantly affect their quality of life.

In some cases, cancer lesions may be localized, allowing for surgical intervention as an effective treatment modality (3). However, in general, for these patients, the indication for radical resection is controversial, as the survival benefits and surgical outcomes have not been clearly determined (6-8).

In this study, we report 3 cases of rectal cancer with perineal skin involvement who underwent multimodality treatment, including effective extended radical resection.

\section{Case report}

Case 1. The patient was a 52-year-old man with rectal cancer that had invaded the perineum. A colostomy was constructed in September, 2003, as the tumor presented with extensive local invasion and complete resection was impossible due to invasion of the pelvic wall. After the operation, the patient received chemotherapy with irinotecan (CPT-11) and doxifluridine and radiotherapy (50 Gy). The patient achieved stable disease, according to Response Evaluation Criteria in Solid Tumor (RECIST); the tumor kept eroding the skin and the patient experienced constant pain. Pelvic computed tomography (CT) revealed that the rectal cancer had invaded the left levator ani and gluteus maximus muscles, perineal skin, bladder, seminal vesicle and prostate gland (Table I, Fig. 1A and B). The lesion was highly advanced locally, but there was no evidence of distant metastasis; therefore, we planned an extended radical resection with curative intent.

We performed a total pelvic exenteration (TPE) with extended resection of adjacent organs, including the perineal skin, in July, 2004 (Fig. 2). To reduce the risk of severe pelvic infection (9), a reconstruction was concomitantly performed, using a vertical rectus abdominis myocutaneous (VRAM) flap. The histological examination of the tumor revealed a 
Table I. Patient profiles.

\begin{tabular}{|c|c|c|c|c|c|}
\hline Cases & $\begin{array}{l}\text { Age/ } \\
\text { gender }\end{array}$ & Stage & Operation & Preoperative treatment & Outcome \\
\hline Case 1 & $52 / \mathrm{M}$ & $\begin{array}{l}\text { T4bN0M0 } \\
\text { stage IIc }\end{array}$ & TPE & $\begin{array}{l}\text { CPT-11 + 5-DFUR and } \\
\text { radiotherapy (50 Gy) }\end{array}$ & $\begin{array}{l}\text { Died } 3 \text { years after } \\
\text { the operation }\end{array}$ \\
\hline Case 2 & $38 / \mathrm{M}$ & $\begin{array}{l}\text { T4bN1bM1a } \\
\text { stage IV }\end{array}$ & TPE & 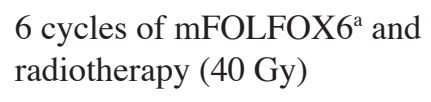 & Alive \\
\hline Case 3 & $50 / \mathrm{F}$ & $\begin{array}{l}\text { T4bN0M0 } \\
\text { stage IIc }\end{array}$ & APR & $\begin{array}{l}\mathrm{UFT} / \mathrm{LV}+\mathrm{CPT}-11 \text { and } \\
\text { radiotherapy (45 Gy) }\end{array}$ & Alive \\
\hline
\end{tabular}

${ }^{a}$ Leucovorin calcium (folinic acid), fluorouracil and oxaliplatin. TPE, total pelvic exenteration; APR, abdominoperineal resection; CPR-11, irinotecan; DFUR, doxifluridine; UFT, uracil/tegafur; LV, leucovorin.
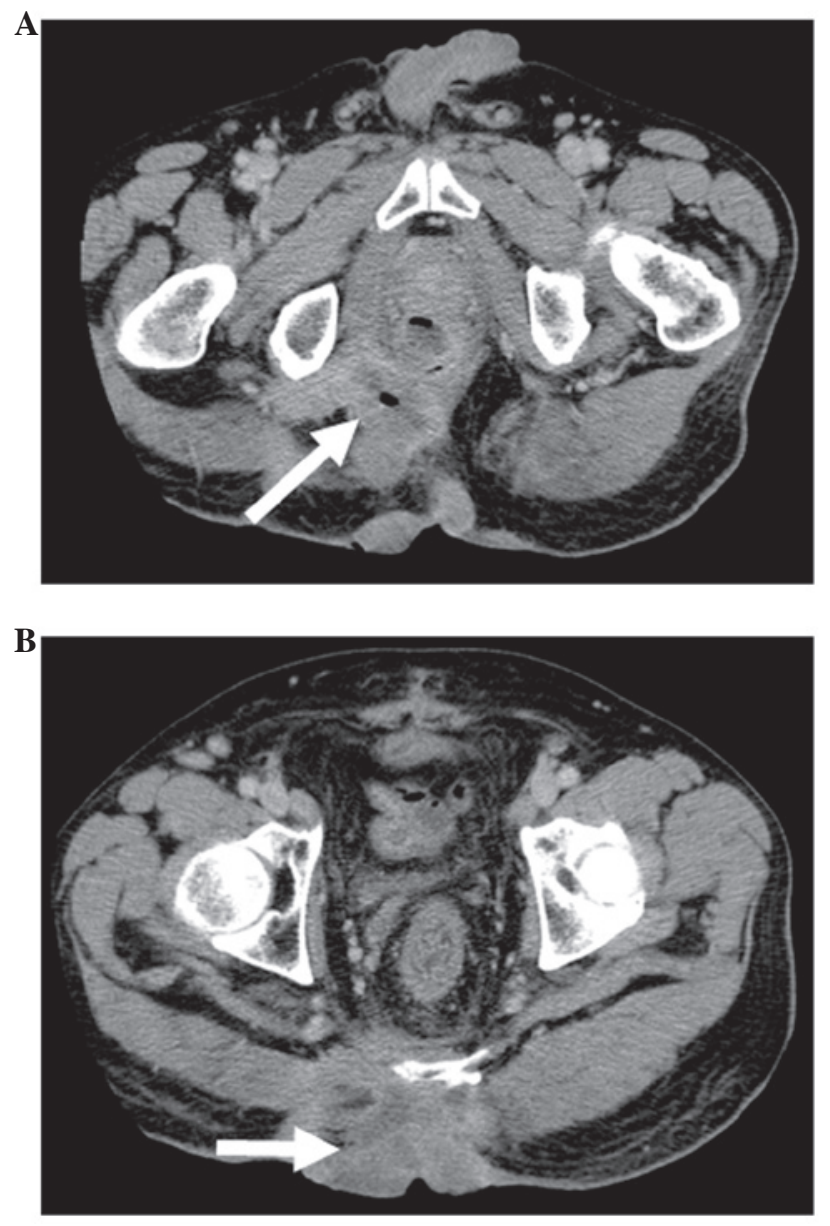

Figure 1. Pelvic CT of rectal cancer prior to total pelvic exenteration surgery. (A) The arrow indicates the site of origin. (B) The arrow indicates the direct invasion of tumor into the skin. CT, computed tomography.

moderately differentiated adenocarcinoma with extensive venous and lymphatic invasion and the examination of the 32 resected lymph nodes confirmed the absence of lymph node metastasis. A histologically complete resection was performed.

The patient succumbed to the disease 3 years after surgery, due to local re-recurrence diagnosed 6 months prior to death. Up to the diagnosis of local re-recurrence, the daily quality of life of the patient was not significantly affected.

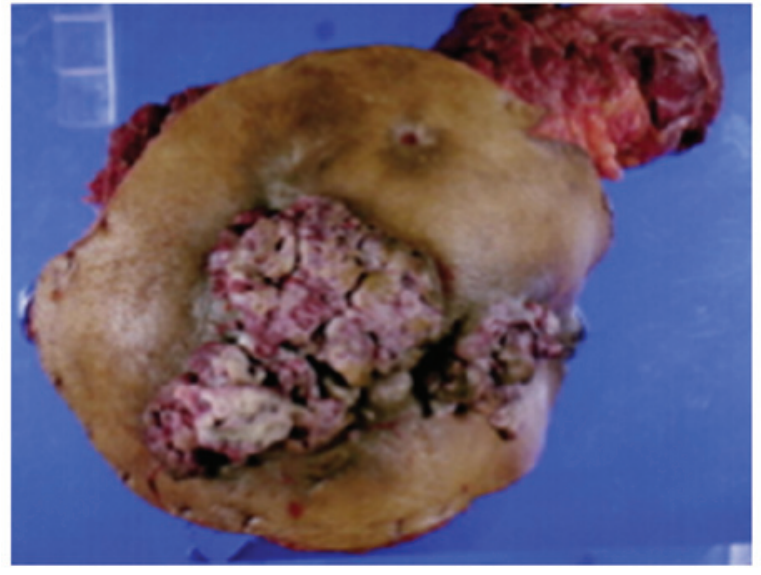

Figure 2. Specimen of total pelvic exenteration surgery, showing skin invasion by rectal adenocarcinoma.

Case 2. The patient was a 38-year-old man, complaining of anal discharge, due to rectal cancer with rectoperineal fistula. The tumor was diagnosed as unresectable, due to a highly advanced primary tumor and the presence of liver metastasis. A colostomy was constructed in December, 2006, prior to the initiation of chemoradiotherapy. The patient received 6 cycles of chemotherapy with mFOLFOX6 and radiotherapy (40 Gy) and achieved a partial response (PR), according to RECIST (Table I).

We performed a TPE on the patient, with extended resection of the perineal skin, followed by reconstruction, using a VRAM flap, in May, 2007 (Fig. 3). The histological examination of the tumor revealed a moderately differentiated adenocarcinoma with extensive venous and lymphatic invasion and the examination of the 38 resected lymph nodes confirmed the absence of lymph node metastasis.

After the operation, the patient received chemotherapy with $\mathrm{mFOLFOX}$ and bevasizumab (Bev), followed by partial hepatectomy. After the hepatectomy, the patient received adjuvant chemotherapy with FOLFIRI and Bev. The patient is currently free from recurrence, 5 years after the hepatectomy.

Case 3. The patient was a 50-year-old woman, complaining of general fatigue and anemia. The patient had a sizeable tumor, visible at the anus (Fig. 4). A rectal biopsy confirmed 


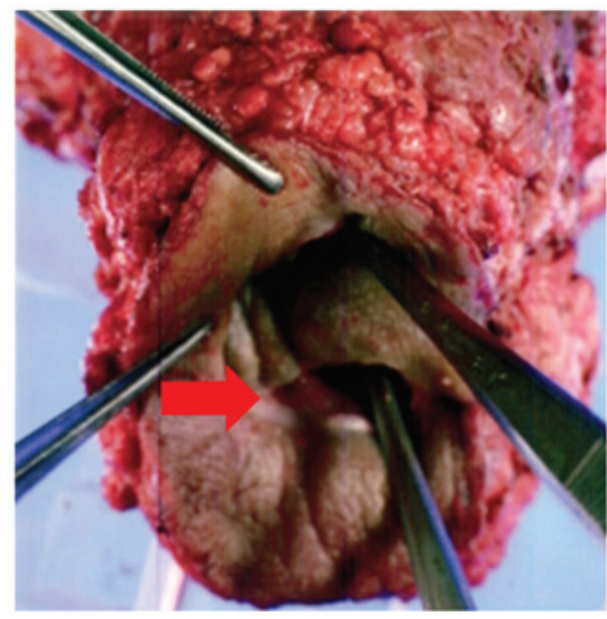

Figure 3. Specimen of total pelvic exenteration surgery. The arrow indicates the rectoperineal fistula.

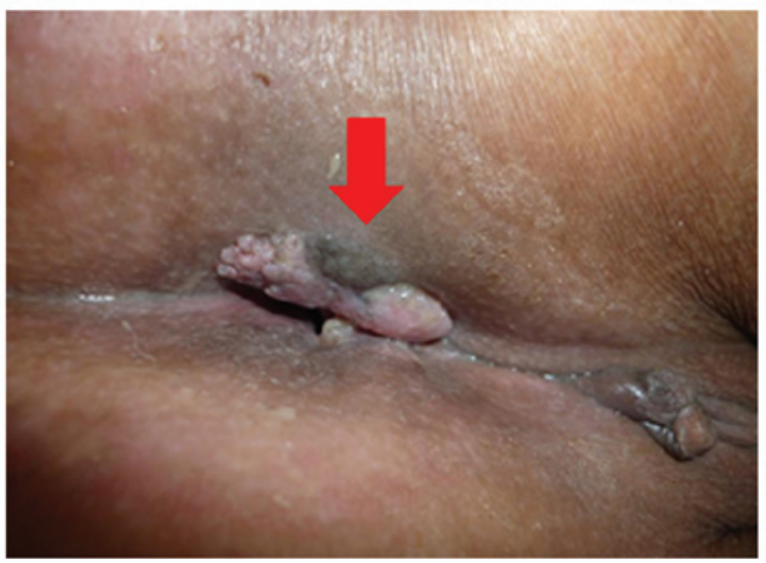

Figure 4. View of the perineal region. The arrow indicates the direct invasion of rectal cancer into the skin.

the diagnosis of poorly differentiated adenocarcinoma. Pelvic magnetic resonance imaging revealed that the rectal cancer had invaded the uterus, cervix, vagina, levator ani muscle and perianal skin (Fig. 5).

The patient received chemotherapy treatment with uracil/tegafur/leucovorin plus CPT-11 and radiotherapy (45 Gy) and achieved a PR, according to RECIST (Table I). We performed abdominoperineal resection with extended resection of the perineal skin, followed by reconstruction using a VRAM flap, in March, 2011. The histological examination of the tumor revealed a poorly differentiated adenocarcinoma with extensive lymphatic invasion and the examination of the 31 resected lymph nodes confirmed the absence of lymph node metastasis.

Seven months after surgery, in October, 2011, the cancer metastasized to the lung. The patient received 3 cycles of chemotherapy with XELOX + Bev, which effectively controlled the metastases. The patient subsequently received a left upper lobectomy in March, 2012, followed by a right S6 segmental resection in June of the same year. The patient experienced local re-recurrence in May, 2013 and is currently under treatment.

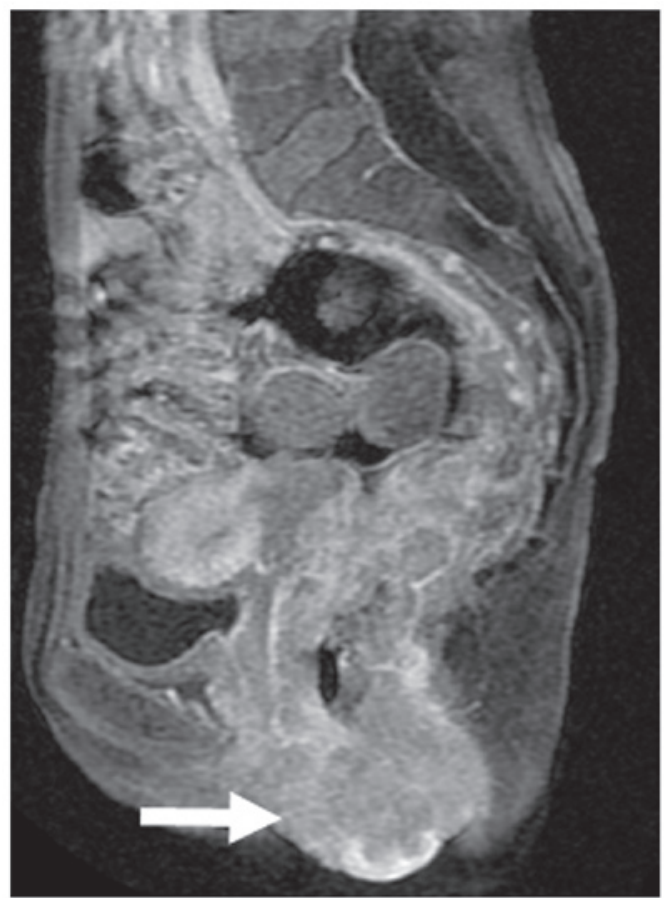

Figure 5. Magnetic resonance imaging of rectal cancer prior to neoadjuvant radiochemotherapy. The arrow indicates the tumor invading into the skin.

\section{Discussion}

Approximately $10 \%$ of patients with colorectal cancer exhibit locally advanced disease with peritoneal involvement (T4a) or invasion of adjacent organs (T4b) at the time of diagnosis (10). In this type of cancer, the most frequently invaded organ is the urinary bladder, followed by the small intestine (11). A literature search revealed that locally advanced rectal cancer directly invading the perineal skin, is rare.

Direct invasion of the tumor into the skin primarily occurs through ulceration or inflammation. Once the malignant ulcers are infected, they are often foul-smelling, painful and bleeding, severely compromising the quality of life of the patients.

According to the National Comprehensive Cancer Network guidelines, neoadjuvant therapy should be administered for the treatment of locally advanced rectal cancer. In addition, it was suggested that extracolonic invasion is not necessarily a poor prognostic factor (11). Rödel et al (12) reported that curative operations may be performed on $94 \%$ of patients with contiguous or adherent-to-adjacent pelvic organs, following CRT with 5-fluorouracil regimen. A resection of the pelvic tumor with negative margins was achieved in $84 \%$ of patients.

Rectal cancer with massive perineal skin invasion should be managed differently from locally advanced rectal cancer, since, once tumor cells have invaded the perineal skin, tumor dissemination may occur via hematogenous and lymphatic routes (13). There are currently no reports on the appropriate treatment for such significantly advanced local rectal cancer.

In our cases, the patients did not achieve a complete cure. All the patients requested a surgical resection as they all suffered from severe and annoying symptoms. Therefore, we planned a multimodality treatment with curative intent, despite the lack of firm evidence. None of the cases had severe postoperative 
complications. The patients almost fully returned to their daily activities. In particular, the multimodal treatment was highly effective in Case 2, although the patient had liver metastasis.

The outcome of these cases shed light on the multimodal treatment, including extended surgery, for rectal cancers with massive perineal skin invasion. There are currently no data on the effectiveness of various treatment modalities for this type of cancer, as there are simply too few cases to make definitive conclusions. An extensive study should be conducted to determine the prognostic factors for long-term survival. Such studies could be informative to the selection of patients who should receive multimodal treatment with curative intent.

In conclusion, for rectal cancer patients with massive invasion to the perineum, multimodal treatment, including extended radical surgery, can be an effective treatment of choice, in terms of balancing cancer control and quality of life.

\section{References}

1. Sgambati SA and Barrows GH: Cutaneous metastasis of colon carcinoma: a case report. Conn Med 57: 665-667, 1993.

2. Goris Gbenou MC, Wahidy T, Llinares K, et al: Atypical phimosis secondary to a preputial metastasis from rectal carcinoma. Case Rep Oncol 4: 542-546, 2011.

3. Lookingbill DP, Spangler N and Sexton FM: Skin involvement as the presenting sign of internal carcinoma-a retrospective study of 7316 cancer-patients. J Am Acad Dermatol 22: 19-26, 1990.
4. Reilly WT, Nelson H, Schroeder G, et al: Wound recurrence following conventional treatment for colorectal cancer. A rare but perhaps underestimated problem. Dis Colon Rectum 39: 200-207, 1996.

5. Jensen SL, Shokouh-Amiri MH, Hagen K, et al: Adenocarcinoma of the anal ducts. A series of 21 cases. Dis Colon Rectum 31: 268-272, 1988.

6. Lo S and Crew J: Penile metastasis from rectal carcinoma. Singapore Med J 45: 299, 2004.

7. Maestro MA, Martinez-Pineiro L, Moreno SS, et al: Penile metastasis of rectal carcinoma. Case report and bibliographic review. Arch Esp Urol 64: 981-984, 2011 (In English, Spanish).

8. Yildirim M, Coskun A, Purten M, et al: A clinical case of the penile metastasis from the rectal carcinoma. Radiol Oncol 44: 121-123, 2011.

9. Uemura M, Ikeda M, Sekimoto M, et al: Prevention of severe pelvic abscess formation following extended radical surgery for locally reccurent of rectal cancer. Ann Surg Oncol 16: 2204-2210, 2009.

10. Lehnert T, Methner M, Pollok A, et al: Multiviscerral resection for locally advanced primary colon and rectal cancer: An analysis of prognostic factors in 201 patients. Ann Surg 235: 217-225, 2002

11. Poeze M, Houbiers JG, van de Velde CJ, et al: Radical resection of locally advanced colorectal cancer. Br J Surg 82: 1386-1390, 1995.

12. Rödel C, Grabenbauer GG, Schick C, et al: Preoperative radiation with concurrent 5 -fluorouracil for locally advanced T4-primary rectal cancer. Strahlenther Onkol 176: 161-167, 2000.

13. Kauffman CL and Sina B: Metastatic inflammatory carcinoma of the rectum: tumor spread by three routes. Am J Dermatopathol 19: $528-532,1997$. 